Brit. J. Ophthal. (1958) 42, 251.

\title{
NEW TECHNIQUE IN CAMPIMETRY*
}

BY

\author{
J. V. SMITH \\ London
}

THE standard method of campimetry, consisting of a black screen 1 or 2 metres square with a fixation spot in the centre and the stimulus carried at one end of a pointer, has many disadvantages but has remained popular owing to its simplicity. The most serious disadvantage is uneven illumination of the screen; others are the tendency for test objects to become soiled, and the confusion produced by movements and reflection of light from the rod carrying the stimulus. Efforts have been made to overcome these difficulties: the stimulus may be held by a magnet behind the screen, or is replaced by a projected spot of light which can be varied in size and intensity. The apparatus described helps to overcome these difficulties (Fig. 1).

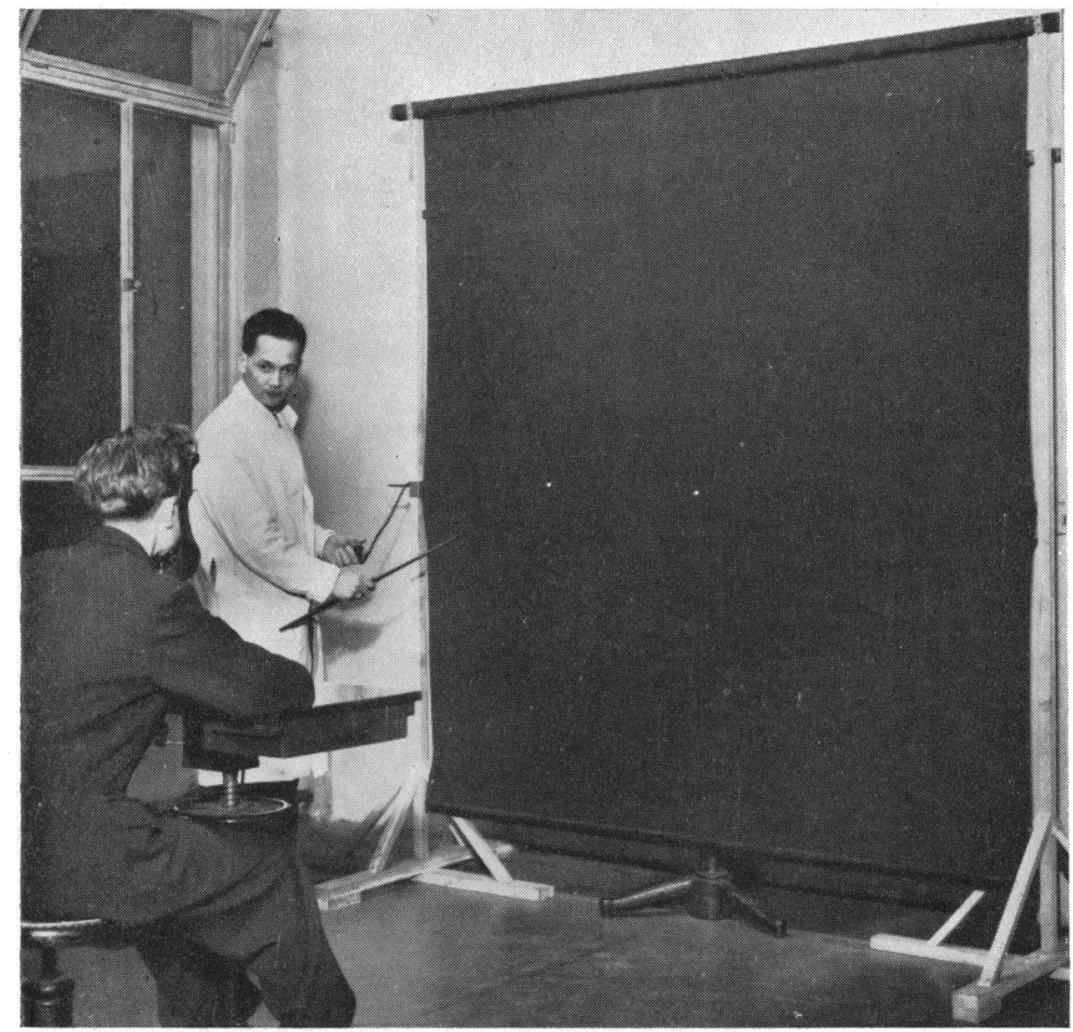

FIG. 1.-Screen and patient in position. The operator controls the light by a switch held in the left hand, and holds the pointer in the right hand.

* Received for publication December 10, 1957. 
The method was devised with the cooperation of Mr. H. C. Weston of the Institute of Ophthalmology, London, and was developed in the Glaucoma Clinic there.

Briefly, it consists of a reversal of the standard method in that the eye follows a moving fixation spot at the end of a pointer. The effective stimulus consists of a light flashing in the centre of the screen, and the light seems to disappear when engulfed by a scotoma.

\section{Apparatus}

The apparatus consists of a light from a suitable source (40-watt frosted bulb) which is made to appear intermittently by being passed through apertures in a rotating disc $D$ (Fig. 2). The motor is so geared that $D$ rotates at 120 r.p.m. In this way the number of flashes per second is directly proportional to the number of sectors cut out in the disc D; two apertures will give four flashes per second and three apertures will give six flashes per second. After experiments carried out subjectively on a number of patients with normal and pathological eyes, it was found that a rate of four flashes per second with a dark period twice as long as the light period was most suitable.
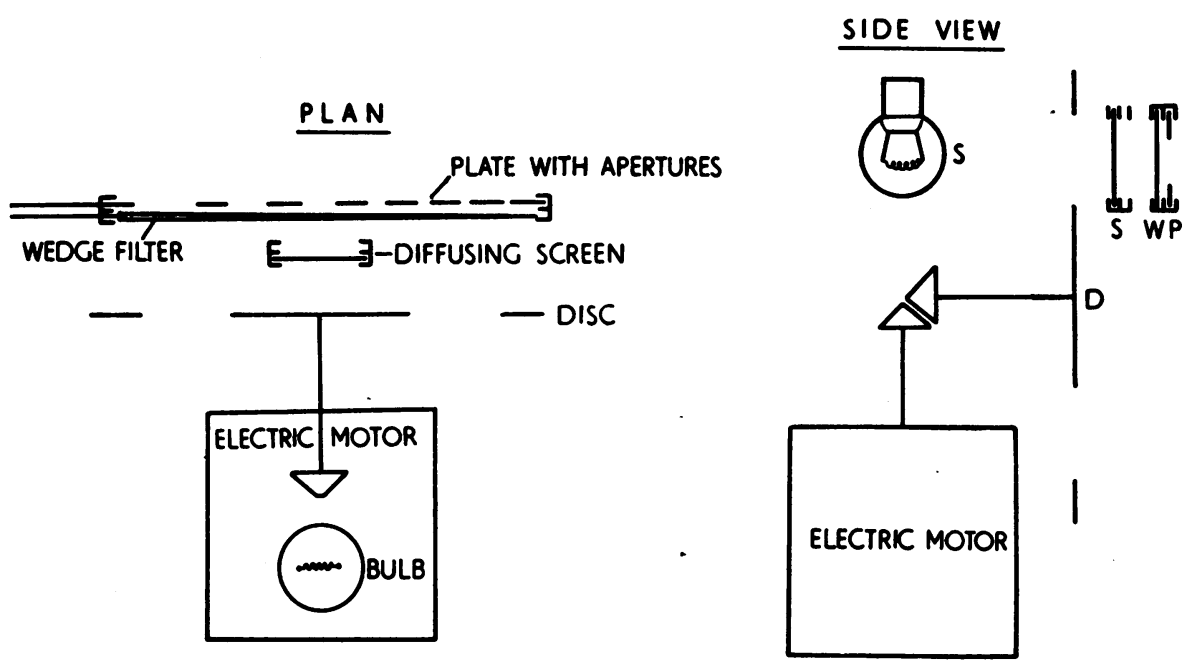

Fig. 2.-Diagram of apparatus.

The intermittent light then passes through a diffusing screen $S$, and its brightness is controlled by a neutral wedge filter W. Finally, a metal plate containing apertures 20-15-10-5-3-2-1 millimetres in diameter controls the size of the flashing light stimulus which replaces the conventional test objects.

\section{Method of Use}

The patient is shown both the white fixation spot and the flashing stimulus. He is instructed to fix and follow the white spot and to tell by any convenient system of verbal or hand signal when the flashing stimulus in the centre 
disappears and when it reappears. The blind spot is usually plotted first with a 10 or 20 millimetre aperture, and then the rest of the field is examined with a 1 or 2 millimetre stimulus.

\section{Interpretation of Results}

A field plotted in this way is the reverse of that obtained with the standard method. The blind spot is on the nasal side and inverted so that any scotoma present will also be inverted. Fig. 3 shows a field of the right eye as charted on the screen. The conventional picture with the blind spot on the temporal side may be obtained by simply rotating Fig. 3 through $180^{\circ}$.

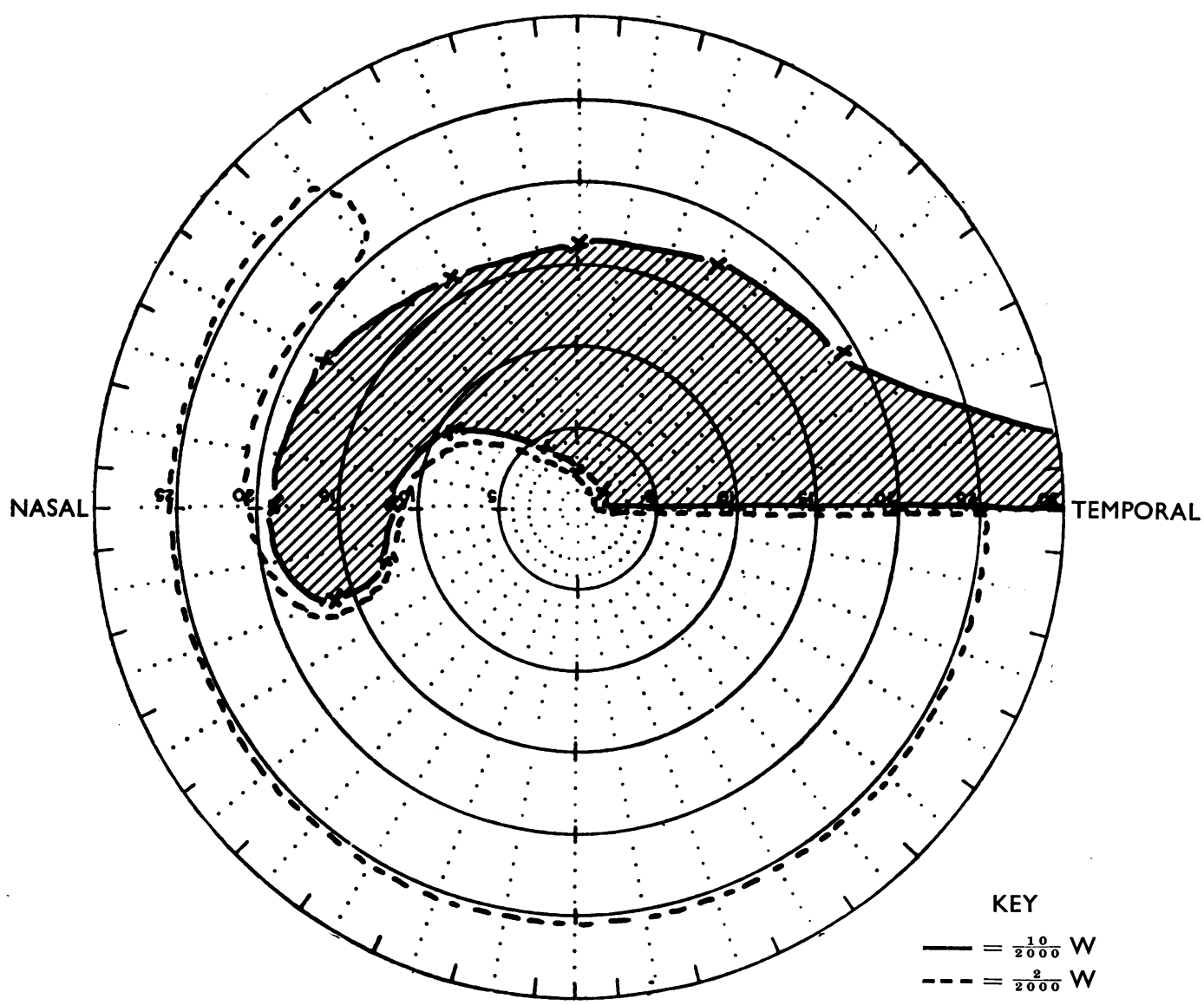

FIG. 3.-Visual field of the right eye as plotted on the screen by the new method.

\section{Advantages}

Uneven illumination over the screen is no longer of primary importance; since the stimulus is stationary its contrast with the background is constant. There is no movement of the stimulus holder to confuse the patient. The 
size, brightness, and colour of the stimulus can be accurately controlled. If the patient is accustomed to wearing a distance correction, this can be worn during the test without interfering with the field of view, since the excursion of the eye needed for the test is well within the boundary of the spectacle frame; the image of the stimulus does not suffer from the distortion which arises in the conventional method because of its oblique position when situated away from the centre of the screen. Such distortions are quite marked when a strong correction is worn. The patient's attention is kept more alert since he has to fix a spot which the examiner is constantly moving about. In the conventional method, with a screen 2 metres square, the stimulus, when placed at the edge of the screen, is further from the eye by a distance of nearly 30 centimetres, so that the visual angle subtended by it is smaller. This effect is further increased by the relatively slit-shaped aperture offered by the pupil at that angle. In this new method the distance between the stimulus and the eye is constant. Lastly, it is of great importance that any method of campimetry should provide some means of concealing the stimulus from the patient's view to check his responses. In the present method this is done by means of a push-button switch which extinguishes the light only and which the examiner can hold in his free hand.

\section{Corrigenda}

In the article entitled "Stereoscopic Vision" by Robert Efron in the December issue (Brit. $J$. Ophthal., 1957, 41, 709), on p. 727 in the last sentence, please read:

In Curve A the average flash duration was $3.5 \mathrm{msec}$., in Curve B it was $42 \mathrm{msec}$., and in Curve $C$ it was $7 \mathrm{msec}$.

On p، 728 , fig. 13 , key, please read:

Curve B $45^{\circ}$ flash of $42 \mathrm{msec}$.

Curve $\mathrm{C}^{\circ}$ flash of $7 \mathrm{msec}$.

It is regretted that it was stated in the review of "The Effect on Binocular Vision of Variations in the Relative Sizes and Levels of Illumination of the Ocular Images", by H. F. Gillott (Brit.J. Ophthal., 1958, 42, 126), that the price was $50 s$. This should read 15s., plus $1 s .6 d$. postage. 